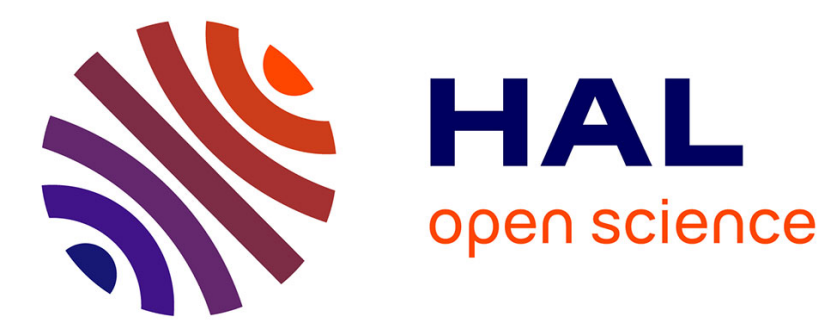

\title{
Optimisation de la chaîne électrique d'un système de micro-cogénération linéaire
}

Marie Ruellan, Thu Thuy Dang, Hamid Ben Ahmed, Laurent Pervond, Pierre Francois, Bernard Multon

\section{- To cite this version:}

Marie Ruellan, Thu Thuy Dang, Hamid Ben Ahmed, Laurent Pervond, Pierre Francois, et al.. Optimisation de la chaîne électrique d'un système de micro-cogénération linéaire. Electrotechnique du Futur 2012, Dec 2011, Belfort, France. pp.11. hal-00665030

\section{HAL Id: hal-00665030 https://hal.science/hal-00665030}

Submitted on 1 Feb 2012

HAL is a multi-disciplinary open access archive for the deposit and dissemination of scientific research documents, whether they are published or not. The documents may come from teaching and research institutions in France or abroad, or from public or private research centers.
L'archive ouverte pluridisciplinaire HAL, est destinée au dépôt et à la diffusion de documents scientifiques de niveau recherche, publiés ou non, émanant des établissements d'enseignement et de recherche français ou étrangers, des laboratoires publics ou privés. 


\title{
Optimisation de la chaîne électrique d'un système de micro- cogénération linéaire
}

\author{
Marie RUELLAN, Thu Thuy DANG, Hamid BEN AHMED, Laurent PREVOND, Pierre FRANCOIS, \\ Bernard MULTON
}

SATIE ENS Cachan

\begin{abstract}
RESUME - Cet article présente une méthodologie d'optimisation d'une chaîne de conversion électrique linéaire (machine à induction contrôlée par convertisseur statique) d'un système de micro-cogénération thermo-mécanoélectrique à moteur Stirling. Dans un premier temps, nous décrivons le principe de fonctionnement du système de micro-cogénération développé dans le cadre du projet CETI (ANR). Les couplages entre les phénomènes thermiques et électriques étant supposés découplés (le couplage mécanique s'effectuant au travers la force de récupération avec une loi de type amortissement visqueux), une description des modèles de la chaîne électrique est proposée. La méthode de son optimisation globale sur cycle est ensuite décrite et l'analyse des résultats obtenus est enfin effectuée.
\end{abstract}

\begin{abstract}
This paper presents an optimization methodology of an electrical conversion chain ( induction machine controled by static converters) for a linear thermo-mecanic-electrical micro-cogeneration system using a Stirling technology. In a first time, the micro-cogeneration system principle is described (developed within the framework of the project CETI (ANR). The thermical and electrical phenomena are supposed decoupled (the mechanical coupling is done through the strength of recovery with a law-like viscous damping), a description of the electrical chain is proposed. The cycle global optimization methodology is presented and the results are then analysed.
\end{abstract}

MOTS-CLES - micro-cogénération, moteur Stirling, optimisation, générateur linéaire à induction, convertisseur statique.

\section{Introduction}

Cette étude concerne un micro-cogénérateur destiné à alimenter en énergie électrique et thermique une habitation individuelle. Il est constitué de deux moteurs d'entraînement de type Stirling associés à un générateur électrique linéaire à induction (figure 1). Pour des raisons d'encombrement, mais aussi, de manière à pouvoir 'encapsuler' l'ensemble dans une enceinte close sans joint, le choix d'une intégration du moteur d'entraînement et du générateur a été fait. Le tout est donc composé d'une chaîne thermo-mécanique couplée à une chaîne électromécanique. L'énergie électrique produite est injectée, via un double convertisseur AC/DC/AC, dans un réseau domestique monophasé $230 \mathrm{~V}-50 \mathrm{~Hz}$.

Les deux moteurs Stirling travaillent en opposition de phase. Les pistons de chacun des moteurs sont liés rigidement. De ce fait ils sont considérés comme un unique piston qui est également le 'mover' du générateur. L’ensemble constitue le moteur Stirling à « piston libre double effet». 


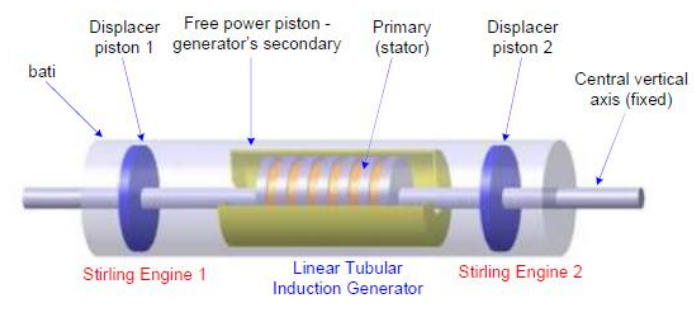

(a)

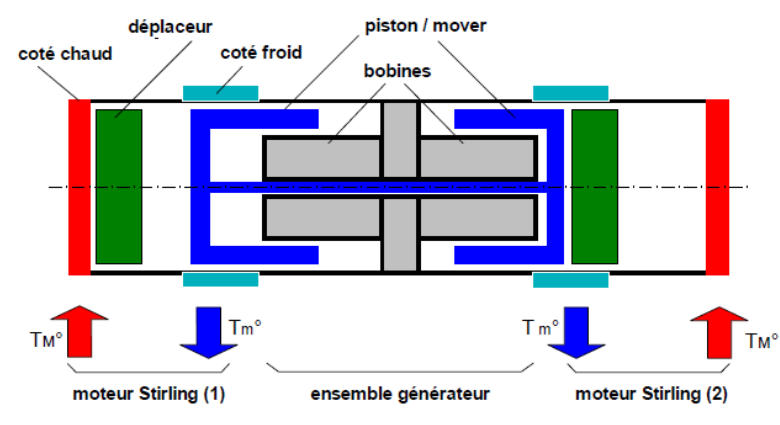

(b)

Figure 1: Schémas du moteur Stirling étudié et de son générateur électromagnétique linéaire à induction.

Le piston commun, ou mover, est entraîné dans un mouvement alternatif selon son axe. Sa course (amplitude et fréquence) dépend de la température de la chambre chaude, de celle de la chambre froide et de la force électromagnétique que la chaîne électrique oppose au moteur. Cette force d'opposition est récupérative de forme et d'amplitude optimisées adaptées au bon fonctionnement de la chaîne thermo-mécanique notamment concernant sa stabilité [1].

Comme nous l'avons déjà précisé, deux moteurs thermiques travaillent en opposition de phase. C'est-à-dire que le temps de détente d'un moteur coïncide au temps de compression de l'autre moteur ou d'une autre manière l'un constitue le "ressort de rappel" de l'autre. Par contre, un tel moteur Stirling n'est pas naturellement stable [1-2]. Cette stabilité devra être assurée par le contrôle/commande de la machine à induction qui est globalement génératrice sur un cycle mais peut fonctionner à certains instants en moteur.

\section{Modèle et méthodologie d'optimisation}

Il s'agit ici de présenter la méthode générale d'optimisation du dimensionnement ainsi que les modèles utilisés. L'outil d'optimisation utilisé est l'algorithme NSGA II [3-4].

L'optimisation concerne uniquement le dimensionnement des éléments de la chaîne de conversion électrique. Celle-ci est présentée sur la figure 2 où l'on distingue le générateur tubulaire linéaire à induction à mover massif (GTLI) en aluminium, deux convertisseurs électroniques de puissance (un triphasé du côté générateur et un monophasé côté réseau) séparés par un bus continu. Le premier convertisseur contrôle la force récupératrice du générateur, le second contrôle la tension du bus DC et s'assure de l'injection au réseau domestique d'un courant sinusoïdal en phase avec la tension (absorption sinusoïdale).

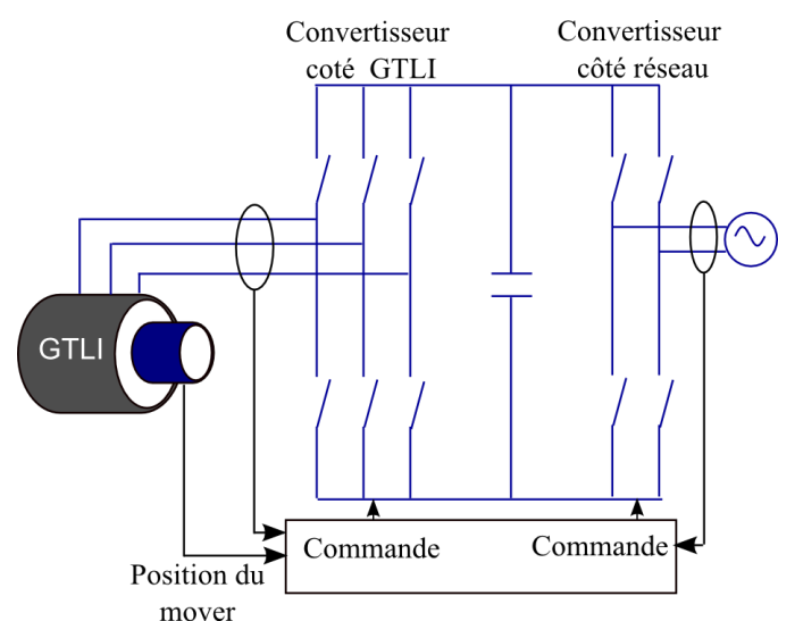

Figure 2 : Schéma de la chaîne de conversion électrique du générateur linéaire tubulaire à induction

Le dimensionnement optimal de la chaine électrique consiste à déterminer les paramètres géométriques du générateur et les calibres courants des interrupteurs de puissance des convertisseurs (sachant que la tension du bus continu est supposée imposée à 400V). Les objectifs choisis sont la minimisation de la masse (parties actives) ainsi que la minimisation de la puissance apparente divisée par le rendement. La figure 3 présente le synoptique d'optimisation. 


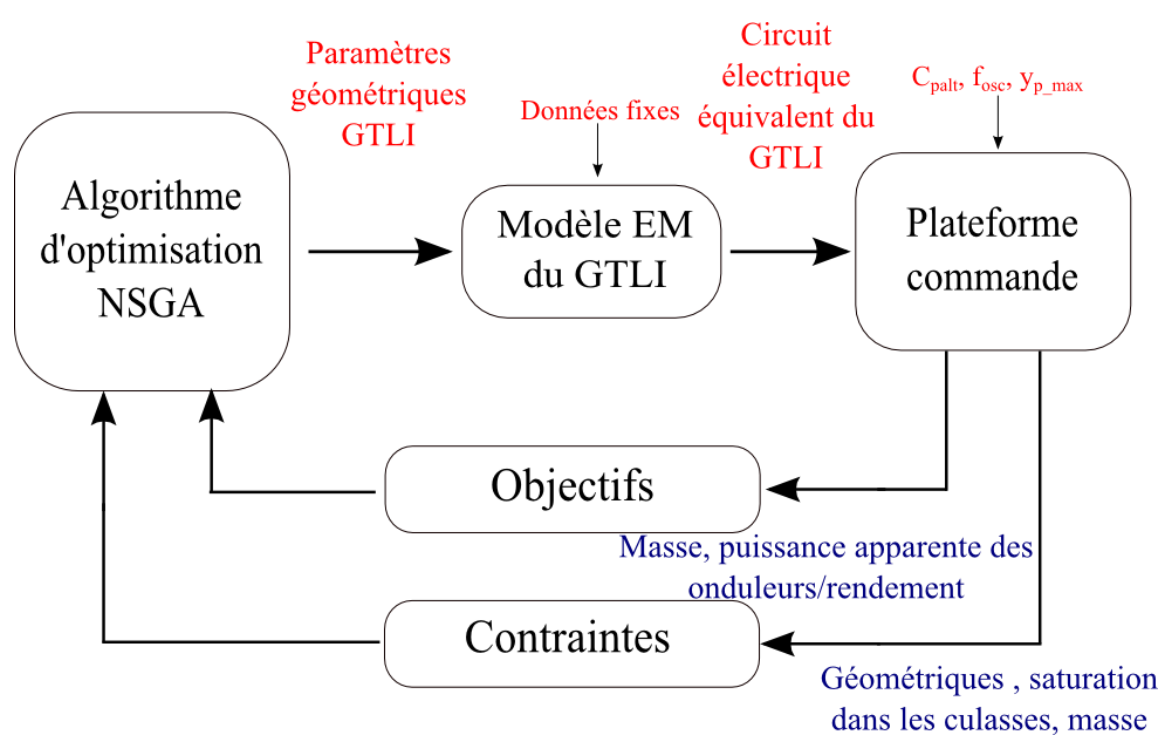

Figure 3 : Synoptique d'optimisation

Nous détaillons dans ce qui suit les différents blocs présents dans le synoptique.

\subsection{Modèle électromagnétique de la machine [3]}

Le modèle électromagnétique du générateur permet de déterminer les valeurs des éléments du schéma électrique équivalent à partir d'une géométrie donnée. Ce calcul est réalisé en utilisant une approche analytique basée sur la résolution formelle des équations de Maxwell. Deux principales hypothèses simplificatrices ont été adoptées : linéarité du circuit magnétique et effets d'extrémités négligés. La démarche est présentée de façon détaillée dans [2].

L'inducteur (stator) est lissé et remplacé par une densité linéique de courant dont l'expression est donnée par :

$$
\lambda_{s}=\sum_{m=1,3, \ldots} \widehat{\lambda_{\mathrm{n}}} e^{j\left(\omega_{s} t-m k z\right)}
$$

$\omega_{\mathrm{s}}$ est la pulsation des courants inducteurs (statoriques), $\mathrm{m}$ est le rang de l'harmonique

L'effet d'encoches est pris en compte au travers un coefficient de Carter d'une part et d'autre part au travers le développement en série de Fourier de la répartition spatiale de la densité de courant (les effets sur les courants induits dans le mover sont donc pris en compte).

Dans ces conditions, on aboutit à la solution suivante du potentiel vecteur donné pour chaque zone $n$ constituant le générateur (cf. figure 5) :

$$
\mathrm{A}^{[\mathrm{n}]}=\sum_{\mathrm{m}}\left[\mathrm{X}_{\mathrm{m}}^{[\mathrm{n}]} \mathrm{I}_{1}\left(\gamma_{\mathrm{m}}^{[\mathrm{n}]} \mathrm{r}\right)\right]+\left[\mathrm{Y}_{\mathrm{m}}^{[\mathrm{n}]} \mathrm{K}_{1}\left(\gamma_{\mathrm{m}}^{[\mathrm{n}]} \mathrm{r}\right)\right] \mathrm{e}^{\mathrm{j}\left(\mathrm{k}_{\mathrm{m}}^{[\mathrm{n}]} \mathrm{z}-\omega_{\mathrm{s}} \mathrm{t}\right)}
$$

Avec :

$\gamma_{m}^{[n]}=\sqrt{\left(k_{m}^{[n]}\right)^{2}+j \omega_{s} g_{m}^{[n]} \mu^{[n]} \sigma^{[n]}}$

$\mathrm{k}_{\mathrm{m} \zeta}^{[\mathrm{n}]}=\frac{\pi}{\tau}\left(m+\zeta \frac{2 \tau}{L}\right)$

$X_{m}^{[n]}$ et $Y_{m}^{[n]}$ représentent des constantes d'intégration calculées à partir des conditions aux limites. $\mathrm{I}_{1}$ et $\mathrm{K}_{1}$ sont des fonctions de Bessel de première et seconde espèce d'ordre $1, \mathrm{~g}^{\mathrm{n}}, \mu^{\mathrm{n}}$ et $\sigma^{[n]}$ représentent respectivement le glissement, la perméabilité magnétique et la conductivité électrique de la zone [n]. Les culasses intérieure et extérieure sont composées de fer et le mover est en aluminium. Les caractéristiques de ces matériaux sont indiquées en annexe 1 . 


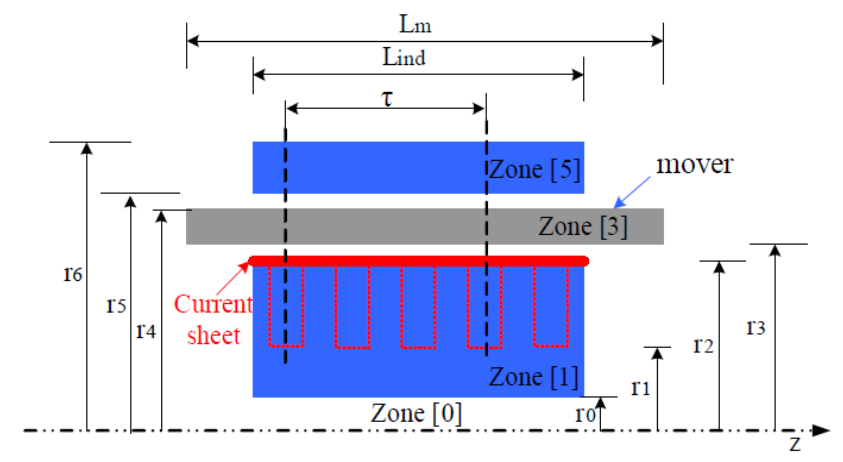

Figure 5: schéma du GTLI

A partir de cette expression, il est aisé de remonter aux expressions des champs magnétiques et électriques :

$\mathrm{H}_{\mathrm{z}}^{[\mathrm{n}]}(\mathrm{z}, \mathrm{r}, \mathrm{t})=\frac{1}{\mu^{[\mathrm{n}]}}\left(\frac{\mathrm{A}^{[\mathrm{n}]}}{\mathrm{r}}+\frac{\partial \mathrm{A}^{[\mathrm{n}]}}{\partial \mathrm{r}}\right)$

$E_{\vartheta}^{[n]}(z, r, t)=j \omega_{s} A^{[n]}$

La puissance peut être déterminée dans chaque zone à l'aide du flux du vecteur de Poynting à travers chaque surface $S^{[n]}$ :

$$
\mathrm{P}^{[\mathrm{n}]}\left(\mathrm{r}^{[\mathrm{n}]}\right)=\iint_{S^{[n]}} \overrightarrow{\mathrm{E}} \wedge \overrightarrow{\mathrm{H}} \mathrm{ds}=\iint_{S^{[n]}} \mathrm{E}_{\theta} \mathrm{H}_{\mathrm{z}} \mathrm{ds}=\frac{1}{2} \mathrm{E}_{\theta}\left(\gamma^{[\mathrm{n}]} \mathrm{r}^{[\mathrm{n}]}\right) \mathrm{H}_{\mathrm{z}}^{*}\left(\gamma^{[\mathrm{n}]} \mathrm{r}^{[\mathrm{n}]}\right) S^{[\mathrm{n}]}
$$

La puissance transmise au mover nous permet de déterminer la force axiale appliquée au mover :

$\mathrm{F}=\frac{\operatorname{Real}\left[\mathrm{P}\left(\mathrm{r}_{3}\right)-\mathrm{P}\left(\mathrm{r}_{2}\right)\right]}{\vartheta_{\mathrm{s}}}$

Où $\vartheta_{\mathrm{s}}=\frac{\tau}{\pi} \omega_{\mathrm{s}}$ est la vitesse de synchronisme

Une comparaison du modèle analytique avec des mesures statiques de force réalisées sur une maquette de démonstration a montré une bonne concordance [2]. Afin d'améliorer le modèle, nous avons développé un modèle 3D tenant compte des effets d'extrémité. Ce modèle a été validé expérimentalement et numériquement. Cependant, lors de l'optimisation, ce modèle complexe s'avère être relativement exigeant en temps de calcul. Nous avons donc fait le choix, dans le cadre de cette pré-optimisation, de nous limiter au modèle simplifié 2D décrit précédemment. Des études fines et des vérifications a postériori pourront être réalisées pour les solutions pré-optimales obtenues.

\subsubsection{Circuit équivalent}

La détermination des courants et tensions, aux bornes des phases du générateur, nécessaires au bon fonctionnement de l'ensemble est réalisée en supposant une commande vectorielle parfaite. Celle-ci nécessite de connaitre le modèle électrique équivalent du générateur. Ce modèle est établi à partir du modèle électromagnétique analytique précédent décrit en supposant que le mouvement du générateur est suffisamment lent permettant de négliger les phénomènes transitoires électromagnétiques d'établissement des courants dans le mover. Par ailleurs, les pertes magnétiques ne sont pas considérées, compte tenu de la difficulté, à ce stade, de les évaluer précisément.

Le modèle est donné à la figure 7 où $\mathrm{L}_{\mathrm{m}}$, représente l'inductance magnétisante. Elle est obtenue à partir du calcul de la puissance réactive magnétisante $\mathrm{Q}_{\mathrm{m}}$. $\mathrm{R}_{\mathrm{r}} / \mathrm{g}$ est la résistance rotorique déterminée à partir de la puissance transmise au secondaire $\mathrm{P}_{\mathrm{tr}} .1_{\mathrm{fr}}$ est l'inductance de fuites rotoriques obtenue à partir de la puissance réactive au secondaire $\mathrm{Q}_{\mathrm{tr}}$. Ces puissances sont calculées à partir du vecteur de Poynting :

$\mathrm{Q}_{\mathrm{m}}=\operatorname{Im}\left[\mathrm{P}\left(\mathrm{r}_{2}\right)\right]$ 


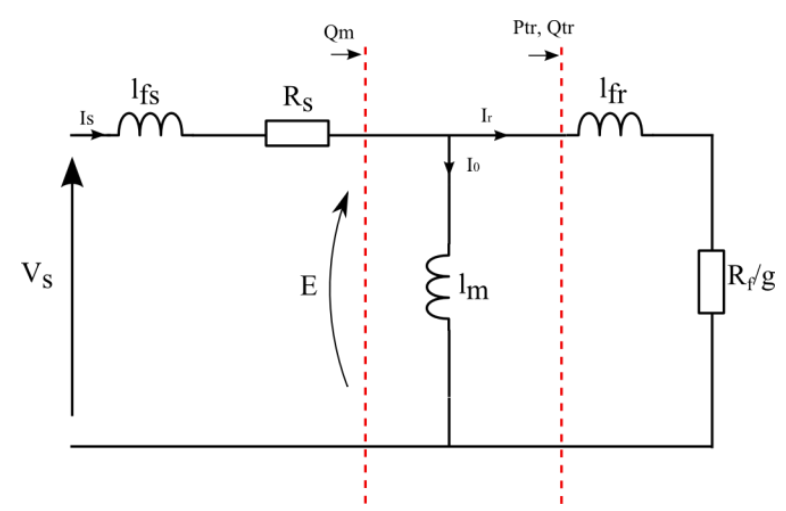

Figure7 : circuit équivalent d'une phase du GTLI.

Ainsi, des expressions ci-dessous on en déduit les valeurs des paramètres du schéma électrique équivalent. Certains paramètres telle que la résistance rotorique dépendent de la fréquence des courants rotoriques et donc du glissement. Pour la phase de dimensionnement, cette dépendance n'est pas prise en compte.

$\mathrm{E}=\frac{\sqrt{\left(\mathrm{P}_{\mathrm{tr}}\right)^{2}+\left(\mathrm{Q}_{\mathrm{m}}+\mathrm{Q}_{\mathrm{tr}}\right)^{2}}}{3 \mathrm{I}_{\mathrm{s}}}$

$\mathrm{L}_{\mathrm{m}}=\frac{3 \mathrm{E}^{2}}{\omega_{\mathrm{s}} \mathrm{Q}_{\mathrm{m}}}$

$\mathrm{I}_{\mathrm{r}}=\frac{\sqrt{\left(\mathrm{P}_{\mathrm{tr}}\right)^{2}+\left(\mathrm{Q}_{\mathrm{tr}}\right)^{2}}}{3 \mathrm{E}}$

$\frac{\operatorname{Rr}}{\mathrm{g}}=\frac{\mathrm{P}_{\mathrm{tr}}}{3\left(\mathrm{I}_{\mathrm{r}}\right)^{2}}$

$\mathrm{l}_{\mathrm{fr}}=\frac{\mathrm{Q}_{\mathrm{tr}}}{3 \omega_{\mathrm{s}}\left(\mathrm{I}_{\mathrm{r}}\right)^{2}}$

De ce schéma on peut déduire un schéma de type bobines couplées dont les paramètres sont $R_{s}, R_{r}, L_{s}, L_{r}$ et $\mathrm{M}$ qui représentent respectivement les résistances d'une phase statorique et rotorique équivalente, les inductances cycliques primaire et secondaire et la mutuelle inductance stator/rotor.

\subsection{Plateforme énergétique}

La plateforme énergétique consiste, pour un dimensionnement donné, à calculer les pertes dans la chaine électrique. Ces pertes sont fonction des courants et tensions dans les divers éléments de la chaine. La démarche est décrite sur le schéma de la figure 9. Différentes hypothèses ont été adoptées afin de simplifier les calculs. Comme mentionné dans le paragraphe précédent, nous supposons que les asservissements sont 'parfaits' et nous raisonnons en valeurs moyennes instantanées.

Les entrées du modèle sont les caractéristiques du moteur Stirling et le profil de déplacement du mover (course $\mathrm{y}_{\mathrm{pmax}}$ et fréquence de battement $\mathrm{f}_{\text {osc }}$ ). Les valeurs de ces paramètres fixes sont données en annexe dans le tableau 3. Le mouvement est naturellement alternatif sinusoïdal car le phénomène thermomécanique est fondamentalement résonnant. Par ailleurs, la forme de la force récupératrice que devra opposer le générateur au moteur Stirling, est imposée sous la forme d'un frottement visqueux de coefficient noté $\mathrm{C}_{\text {palt }}$. La figure 8 présente un exemple de variation de la force et de la vitesse de translation du mover.

$\mathrm{F}_{\text {gene }}=-\mathrm{C}_{\text {palt }} \frac{\mathrm{dyp}}{\mathrm{dt}}$

Avec :

$y_{p}(t)=y_{p \max } \cos \left(2 \pi f_{o s c} t\right)$ 


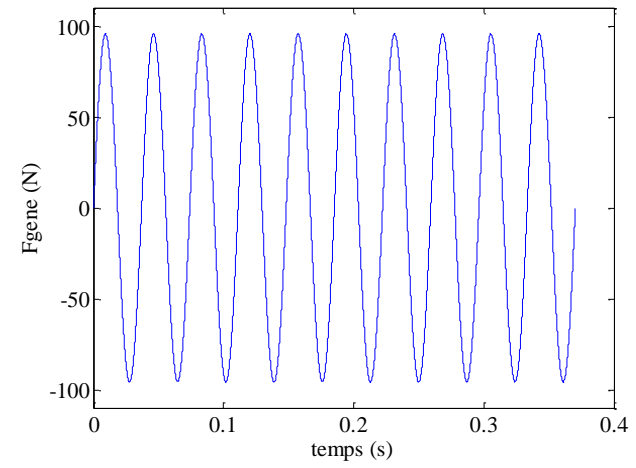

(a)

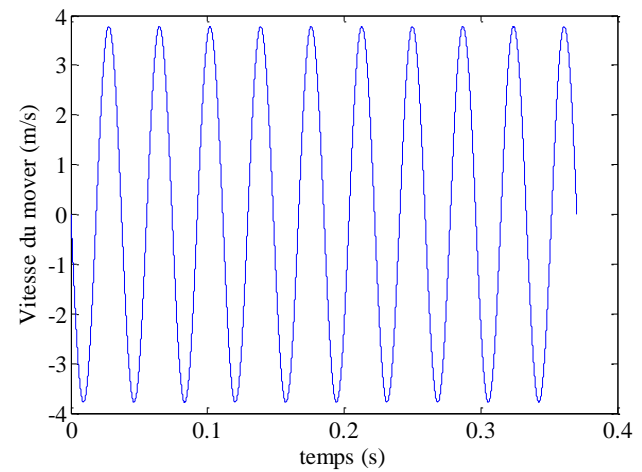

(b)

Figure 8 : Allures de la force récupératrice $F_{\text {gene }}(a)$ et de la vitesse de déplacement du mover (b)

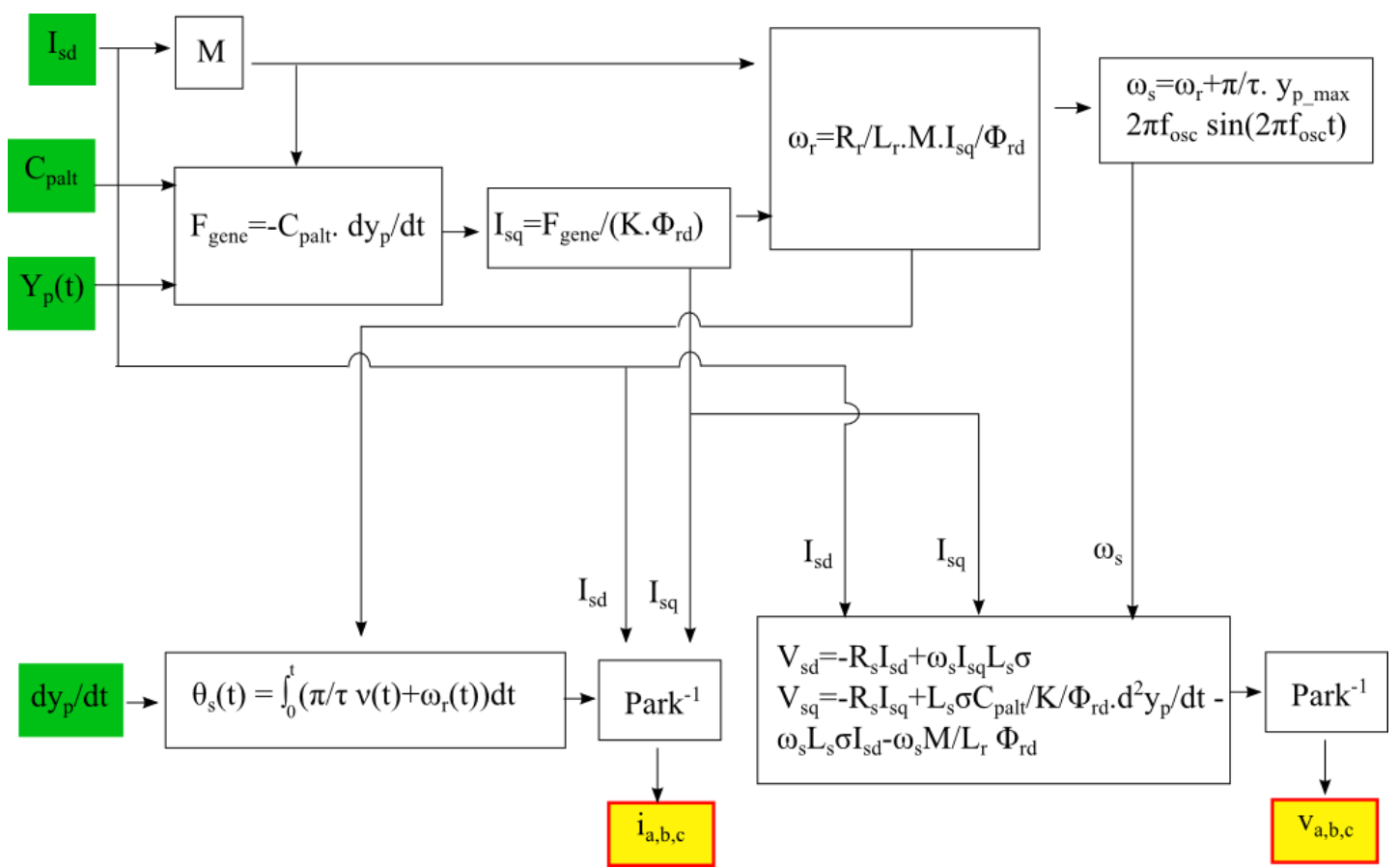

Figure 9 : Schéma de la plateforme énergétique (en vert les données, en jaune les sorties du modèle)

Une fois les calculs des courants et tensions dans le TLIG déterminés, il s'agit maintenant de calculer les courants traversant les divers interrupteurs de puissance ainsi que la capacité de filtrage. Ce calcul est réalisé en valeur moyenne instantanée à l'échelle de la période de découpage. Pour le convertisseur côté réseau, la forme sinusoïdale du courant est imposée par un asservissement supposé parfait. Les pertes par commutation et par conduction dans les interrupteurs sont calculées analytiquement à l'aide de [8].

Compte tenu du fait que les deux convertisseurs électroniques ne sont pas soumis aux mêmes variations de courants, il est important de distinguer les pertes du convertisseur triphasé côté générateur de celui monophasé côté réseau. Ces pertes de commutation $\left(\mathrm{P}_{\mathrm{com}}\right)$ et de conduction $\left(\mathrm{P}_{\text {cond }}\right)$ s'expriment à partir notamment des caractéristiques des interrupteurs commandés (IGBT, indice T) et des diodes (indice D). Ainsi, pour le convertisseur côté générateur, nous ne pouvons pas utiliser des formulations classiques valables uniquement dans au cas sinusoïdal, un calcul précis des courants moyens et efficaces doit donc être au préalable effectué :

$$
\begin{aligned}
& P_{\text {cond }}=V_{\text {CEO }} \sum_{n=1}^{6} I_{T_{\text {moy }(n)}}+R_{\text {ds Oo }}\left[\sum_{n=1}^{6}\left(I_{T_{\text {eff }(n)}}\right)^{2}\right]+V_{D O} \sum_{n=1}^{6} I_{D_{\text {moy }(n)}}+R_{D}\left[\sum_{n=1}^{6}\left(I_{D_{\text {eff }(n)}}\right)^{2}\right] \\
& P_{\text {com }}=2 \frac{V_{\text {bus }}}{V_{\text {test }}} f_{\text {dec }}\left[\left(\sum_{n=1}^{6} I_{T_{\text {moy }(n)}}+I_{D_{\text {moy }(n)}}\right)\left(b_{\text {on }}+b_{\text {off }}\right)+\frac{1}{2}\left(a_{\text {on }}+a_{\text {off }}\right)\right]
\end{aligned}
$$


Concernant le convertisseur côté réseau, les courants et tensions sont sinusoïdaux et en phase, nous pouvons donc appliquer des formulations classiques des pertes faisant intervenir uniquement la valeur maximale du courant côté réseau :

$$
\begin{aligned}
& P_{\text {cond }}=\frac{2 V_{C E O} I_{\max }}{\pi}\left(1+\frac{\pi}{4 m_{a}}\right)+4 R_{\text {dson }} \frac{I_{\text {max }}^{2}}{8}\left(1+\frac{8}{3 \pi m_{a}}\right)+\frac{2 V_{D 0} I_{\max }}{\pi}\left(1-\frac{\pi}{4 m_{a}}\right)+4 R_{D} \frac{I^{2} \max }{8}\left(1-\frac{8}{3 \pi m_{a}}\right) \\
& P_{\text {com }}=4 \frac{V_{\text {bus }}}{V_{\text {test }}} f_{\text {dec }}\left[\frac{I_{\text {max }}}{\pi}\left(b_{\text {on }}+b_{\text {off }}\right)+\frac{1}{2}\left(a_{\text {on }}+a_{\text {off }}\right)\right]
\end{aligned}
$$

Où $\mathrm{m}_{\mathrm{a}}$ correspond au facteur de modulation égal au rapport de la tension maximal alternative et de la demie tension du bus DC.

Pour chaque calibre courant proposé par l'optimisation, les paramètres $V_{C E O}, V_{D O}, R_{D}, R_{d s ~ o n}, a_{o n}, b_{o n}, a_{\text {off }}, a_{o f f}$, sont identifiés sur la base des données constructeur.

Les pertes Joule statoriques du générateur sont données par :

$\mathrm{P}_{\mathrm{Js}}=\mathrm{R}_{\mathrm{s}}\left(\mathrm{i}_{\text {aeff }}^{2}+\mathrm{i}_{\mathrm{b} \text { eff }}^{2}+\mathrm{i}_{\mathrm{c} \mathrm{eff}}{ }^{2}\right)$

Les pertes Joule rotoriques sont données par :

$$
\mathrm{P}_{\mathrm{J}_{\mathrm{r}}}=3 \mathrm{R}_{\mathrm{r}}\left(\mathrm{I}_{\mathrm{r}_{-} \text {eff }}^{2}\right) \text { avec } \operatorname{ir}(\mathrm{t})=\sqrt{\mathrm{i}_{\mathrm{rd}}^{2}(\mathrm{t})+\mathrm{i}_{\mathrm{rq}}^{2}(\mathrm{t})}
$$

\subsection{Méthodologie d'optimisation}

Les paramètres à optimiser sont le rayon $\mathrm{r}_{1}$, la longueur et l'épaisseur du mover, la longueur du pas polaire et le nombre de paires de pas polaires, la hauteur des encoches, le nombre de spires par bobine et par phase, l'épaisseur de la culasse extérieure ainsi que les calibres courant côté générateur et côté réseau (figures 3 et 4) [Tableau 2].

Tableau 2. Paramètres d'optimisations et valeurs limites.

\begin{tabular}{lccc} 
Paramètres d'optimisation & Nomination & $\begin{array}{c}\text { Valeurs } \\
\text { mini }\end{array}$ & $\begin{array}{c}\text { Valeurs } \\
\text { max. }\end{array}$ \\
\hline Rayon $\mathrm{r}_{1}(\mathrm{~m})$ & $\mathrm{r}_{1}$ & 0,010 & 0,050 \\
\hline Longueur du mover $(\mathrm{m})$ & $\mathrm{L}_{\mathrm{mov}}$ & 0,005 & 0,500 \\
\hline Epaisseur du mover $(\mathrm{m})$ & $\mathrm{E}_{\mathrm{mov}}$ & 0,001 & 0,500 \\
\hline Pas polaire $(\mathrm{m})$ & $\tau$ & 0,050 & 0,500 \\
\hline Nombre de paires de pas polaires & $\mathrm{N} \tau$ & 1 & 1000 \\
\hline Hauteur des encoches $(\mathrm{m})$ & $\mathrm{H}_{\mathrm{enc}}$ & 0,001 & 0,500 \\
\hline Nombre de bobines par bobine et par phase & $\mathrm{n}_{\text {spires }}$ & 1 & 1000 \\
\hline Hauteur de la culasse extérieure $(\mathrm{m})$ & $\mathrm{E}_{\mathrm{c} \_ \text {ext }}$ & 0,001 & 0,500 \\
\hline Courant nominal du convertisseur géné. $(\mathrm{A})$ & $\mathrm{I}_{\mathrm{kMax} \mathrm{g}}$ & 1 & 12 \\
\hline Courant nominal du convertisseur réseau. $(\mathrm{A})$ & $\mathrm{I}_{\mathrm{kMax} \mathrm{r}}$ & 1 & 12
\end{tabular}

Les deux critères $\mathrm{C}_{1}$ et $\mathrm{C}_{2}$ à minimiser sont :

- la masse des parties actives calculée à partir des volumes de chaque zone et des masses volumiques des matériaux associés :

$\mathrm{C}_{1}=\mathrm{M}_{\mathrm{a}}=\sum_{\mathrm{k}} \mathrm{m}_{\mathrm{vk}} \mathrm{v}_{-} \mathrm{k}$

- le ratio surface silicium/rendement. Ce second critère traduit le coût des convertisseurs pondéré par les pertes de la chaîne électrique. La puissance apparente est calculée en sommant les puissances apparentes des deux onduleurs. Le rendement est le rapport de la puissance en sortie côté réseau sur la puissance mécanique :

$$
\mathrm{C}_{2}=\frac{\mathrm{S}_{\text {conv }}}{\eta_{\text {chaine }}}=\frac{\sum_{\mathrm{k}=1}^{3} \mathrm{v}_{\text {keff }} \mathrm{I}_{\text {keff }}+\mathrm{V}_{\text {reff }} \mathrm{I}_{\text {reff }}}{1-\frac{\sum \text { pertes }}{P_{\text {méca }}}}
$$


Le problème est contraint et l'optimisation doit répondre aux contraintes suivantes :

a. Contraintes géométriques :

a. La masse totale des parties actives doit être inférieure à $5 \mathrm{~kg}$

b. Le rayon extérieur est limité à $0,25 \mathrm{~m}$.

b. Contraintes magnétiques

Compte tenu de l'hypothèse de non-saturation, les inductions magnétiques dans les culasses intérieure et extérieure sont bornées :

$\frac{\phi_{\mathrm{e}}}{\pi\left(\mathrm{r}_{1}^{2}-\mathrm{r}_{0}^{2}\right)}-\mathrm{B}_{\text {sat }} \leq 0$

$\frac{\phi_{\mathrm{e}}}{\pi\left(\mathrm{r}_{6}^{2}-\mathrm{r}_{5}^{2}\right)}-\mathrm{B}_{\text {sat }} \leq 0$

Avec $\mathrm{B}_{\text {sat }}=1,6 \mathrm{~T}$

Le flux $\phi_{\mathrm{e}}$ est déduit du schéma électrique équivalent de la figure 7.

c. Contraintes onduleurs

Nous vérifions que les courants dans l'onduleur côté réseau et dans l'onduleur côté générateur ne dépassent pas les calibres courant associés :

$\left\{\begin{array}{cc}\max \left(\mathrm{I}_{\mathrm{IGBT}}, \mathrm{I}_{\mathrm{D}}\right)_{\text {gene }} \leq \mathrm{I}_{\mathrm{kMax}} \mathrm{g} \\ \max \left(\mathrm{I}_{\mathrm{IGBT}}, \mathrm{I}_{\mathrm{D}}\right)_{\text {reseau }} \leq \mathrm{I}_{\text {kMax r }}\end{array}\right.$

\section{Résultats d'optimisation}

Nous présentons à la figure 10 les résultats obtenus sous forme de front de Pareto. L'exemple traité correspond à une puissance mécanique sur l'axe du mover de $181 \mathrm{~W}$ (force maximale de $95.7 \mathrm{~N}$, vitesse maximale $3.8 \mathrm{~m} / \mathrm{s}$ ) et une fréquence mécanique d'oscillation de $27 \mathrm{~Hz}$. Nous observons que les deux objectifs sont contradictoires. Les solutions non dominées correspondent donc à un ensemble de compromis entre le ratio puissance apparente/rendement et la masse des parties actives. Ces résultats sont les premiers résultats d'optimisation effectués sur ce problème, d'autres objectifs pourront être choisis par la suite.

A l'analyse des résultats de cette première optimisation, nous constatons que le nombre de paires de pôles (ou le nombre de pas polaires) le long du front de Pareto est toujours minimisé (2 pas polaires). En effet, à l'inverse des actionneurs synchrones notamment à aimants où la réduction du pas polaire engendre une diminution de la masse sans dégrader les performances électromagnétiques de l'actionneur, pour les actionneurs à induction, la réduction du pas affecte très sensiblement les performances.

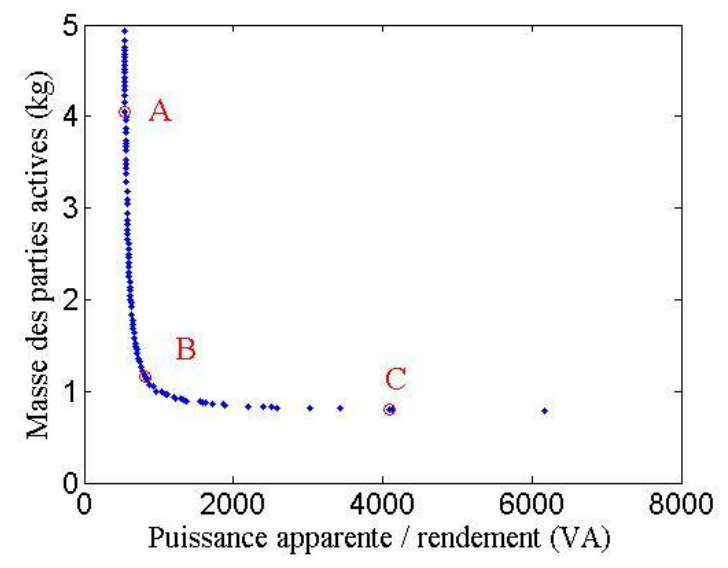

Figure 10 : Front de Pareto d'optimisation dans le plan des objectifs

Les résultats sont détaillés dans le tableau ci-dessous pour les trois configurations distinctes $\mathrm{A}, \mathrm{B}$ et $\mathrm{C}$. La géométrie de chaque configuration est précisée à la figure 11. 


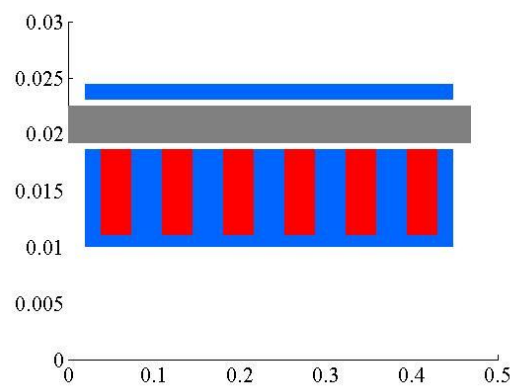

Machine A

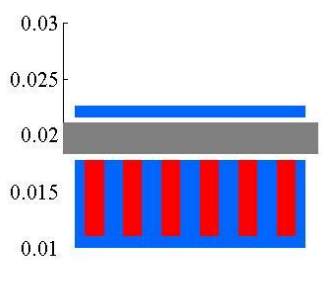

0.005

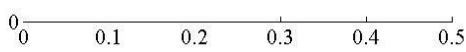

Machine B

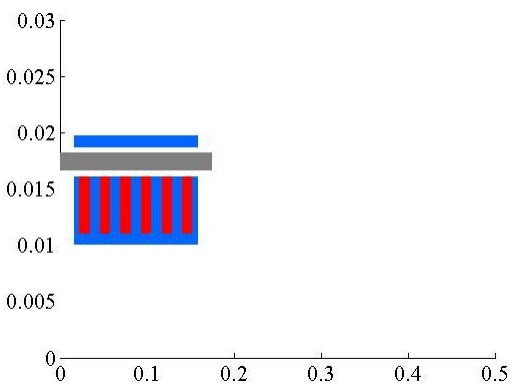

Machine C

Figure 11 : Dessins des machines A, B et C (les dimensions sont indiquées en mètre - échelles différentes en abscisse et en ordonnées)

Tableau 2. Dimension des trois générateurs.

\begin{tabular}{|c|c|c|c|}
\hline Désignation & Machine A & Machine B & Machine C \\
\hline Rayon du fond d'encoches (mm) & 11 & 11 & 11 \\
\hline Longueur du mover (mm) & 469 & 237 & 175 \\
\hline Epaisseur du mover (mm) & 3 & 2 & 1 \\
\hline Hauteur des encoches (mm) & 7 & 5 & 5 \\
\hline Pas polaire $(\mathrm{mm})$ & 214 & 98 & 70 \\
\hline Nombre de paires de pas polaires & 1 & 1 & 1 \\
\hline Epaisseur de la culasse extérieure (mm) & 1 & 1 & 1 \\
\hline Calibre courant de l'onduleur coté génératrice (A) & 6 & 6 & 6 \\
\hline Calibre courant calibre de l'onduleur coté réseau (A) & 2 & 1 & 1 \\
\hline Nbre de spires par bobine et par phase & 50 & 52 & 52 \\
\hline Longueur de l'inducteur (mm) & 428 & 196 & 142 \\
\hline \multicolumn{4}{|l|}{ Objectifs d'optimisation } \\
\hline Puissance apparente totale des 2 convertisseurs (VA) & 297,7 & 238,3 & 150,1 \\
\hline Rendement global sur cycle $(\%)$ & 54 & 29 & 3 \\
\hline Masse des parties actives (kg) & 4,05 & 1,16 & 0,80 \\
\hline Puissance électrique moyenne récupérée (W) & 97,8 & 53,3 & 6,6 \\
\hline \multicolumn{4}{|l|}{ Eléments du schéma électrique équivalent } \\
\hline $\mathrm{Lm}(\mathrm{mH})$ & 45 & 26 & 20 \\
\hline $\operatorname{Lr}(\mathrm{mH})$ & 45 & 27 & 22 \\
\hline $\operatorname{Rs}(\Omega)$ & 0,09 & 0,31 & 0,42 \\
\hline $\operatorname{Rr}(\Omega)$ & 0,33 & 0,73 & 1,28 \\
\hline $\mathrm{M}(\mathrm{mH})$ & 45 & 30 & 20 \\
\hline
\end{tabular}

Concernant la «configuration compromis » choisie (solution B), elle présente une masse active de la génératrice de $1.16 \mathrm{~kg}$, une puissance apparente totale des convertisseurs de $238.3 \mathrm{VA}$ et génère au niveau du réseau une puissance électrique moyenne de $53.3 \mathrm{~W}$. Le rendement global sur cycle de la chaîne électrique obtenu pour cette configuration est de $29 \%$. Les amplitudes des courants triphasés côté générateur sont importantes comme illustré sur la figure 12a. La puissance apparente du convertisseur côté générateur est donc élevée nécessitant un calibre courant de $6 \mathrm{~A}$ sous $400 \mathrm{~V}$ DC. A l'inverse, côté réseau, du fait de la présence de la capacité de filtrage, l'amplitude du courant associé est réduite tel que montré par la figure 12b ce qui entraine une puissance apparente du convertisseur monophasé plus faible (calibre courant 1A, sous 400V DC), En effet, les fluctuations de puissance générée par le générateur sont atténuées par la capacité de filtrage du bus DC entrainant une fluctuation de sa tension dans une plage prédéterminée.

Notons la valeur du pas polaire pour les trois configurations indiquées (variant de 21 à $7 \mathrm{~cm}$ ) qui est sensiblement supérieur à la course fixée du mover qui elle n'est que de $2.2 \mathrm{~cm}$ d'où la forme de variation des courants dans le générateur. 
Enfin, il est intéressant de remarquer sur la figure 13 que le générateur fonctionne sur un cycle globalement en mode générateur mais qu'à des instants courts, proches de la vitesse nulle, il fonctionne en mode frein dissipatif. En effet, pendant ces laps de temps, la machine à induction absorbe de la puissance mécanique fournie par le moteur Stirling et absorbe également de la puissance électrique provenant du bus DC.

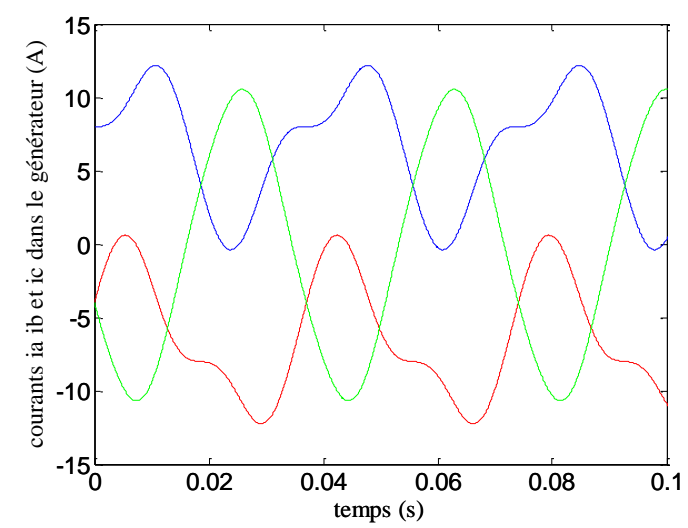

(a)

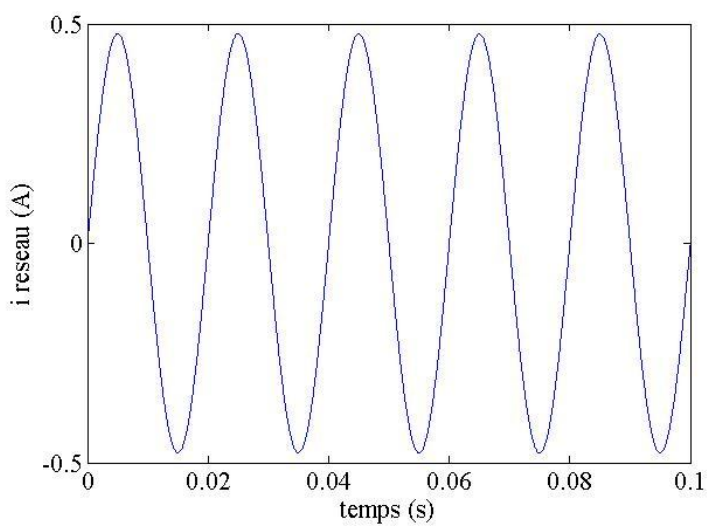

(b)

Figure 12 : Courants au niveau des 3 phases du générateur (a) et au niveau du réseau (b) pour la configuration compromis (solution B)

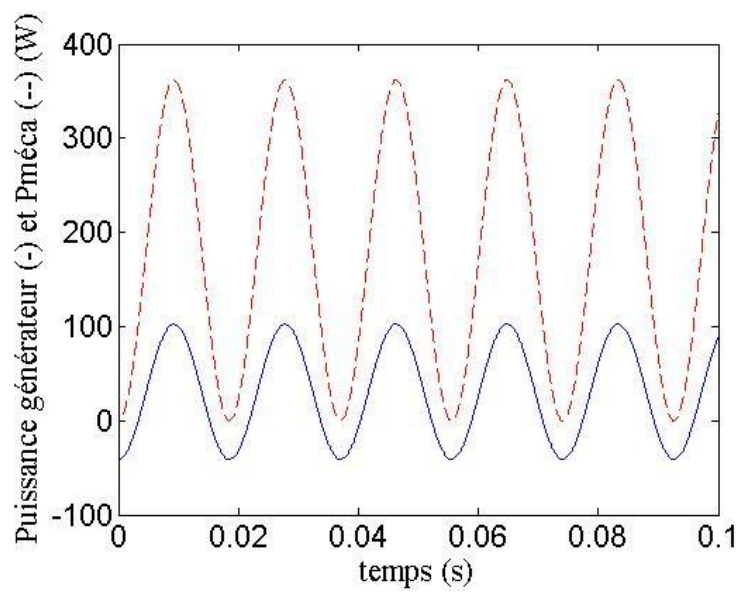

Figure 13 : Puissances électrique générateur et mécanique pour la configuration compromis (solution B)

\section{Conclusion}

Nous présentons dans ce papier une méthodologie d'optimisation de la chaîne électrique linéaire d'un microco-générateur utilisant un moteur Stirling. Les deux objectifs choisis (masse des parties actives, puissances apparentes convertisseurs / rendement) étant contradictoires, les fronts de Pareto obtenus permettent de déterminer un ensemble de solutions optimales.

Ces premiers résultats nous ont permis d'avoir un premier pré-dimensionnement de la chaîne de conversion électrique. Des améliorations pourront être apportées : le modèle du générateur devra par la suite tenir compte des effets d'extrémité ; le coefficient de Carter devra être exprimé en fonction de la géométrie de la machine. Une validation avec un modèle éléments finis pourra également être effectuée sur quelques solutions optimales. Enfin, les pertes magnétiques ont été négligées dans l'évaluation du rendement. Un modèle adéquat devra être construit. L'intégration de ce modèle dans l'optimisation permettra, au-delà d'une meilleure évaluation des critères, de mieux apprécier les épaisseurs optimales des culasses magnétiques.

Concernant les objectifs d'optimisation, d'autres critères peuvent être considérés notamment le coût financier ou énergétique du système (générateur+onduleurs) vs le gain financier ou énergétique lié à l'énergie électrique récupérée au réseau. Le condensateur du bus DC devra être pris en compte dans ces critères d'optimisation.

Le choix d'un générateur de type à induction (GTLI) a été essentiellement dicté par l'intérêt d'avoir une masse en mouvement très faible (ici un mover en aluminium) et un faible impact de la contrainte thermique 
sur le générateur compte tenu de sa proximité avec le Stirling. Une comparaison avec une autre architecture de générateur devra cependant être réalisée. Nous pensons à un générateur à aimants permanents présentant par exemple le même inducteur que celui du GTLI. Le mover en aluminium serait remplacé par un cylindre magnétique dont la surface intérieure comporterait des aimants alternés à aimantation radiale. La distribution axiale et orthoradiale des aimants pourrait être étudiée afin de minimiser l'effort de détente (aimants-denture) nuisible au fonctionnement du moteur Stirling.

\section{Annexe}

Tableau 2. Caractéristiques des matériaux.

\begin{tabular}{lcc} 
Matériau & Caractéristiques & Valeur \\
\hline \hline Perméabilité magnétique du fer & $\mu$ & 5000 \\
\hline Conductivité de l'aluminium & $\sigma$ & $3510^{6}$
\end{tabular}

Tableau 3. Paramètres fixes du problème

\begin{tabular}{lc} 
Désignation & Valeur \\
\hline \hline Fréquence mécanique d'oscillation $\mathrm{f}_{\mathrm{osc}}(\mathrm{Hz})$ & 27 \\
\hline Coefficient de frottements visqueux $\mathrm{C}_{\mathrm{palt}}$ & 25,3 \\
\hline Course du mover $\mathrm{Y}_{\mathrm{pmax}}(\mathrm{m})$ & $2,2310^{-2}$ \\
\hline Rayon de l'axe amagnétique $\mathrm{r}_{0}(\mathrm{~m})$ & $1010^{-3}$ \\
\hline Coefficient de carter & 1,8 \\
\hline Tension du bus DC $(\mathrm{V})$ & 400 \\
\hline Entrefer mécanique $(\mathrm{mm})$ & 0,5 \\
\hline Vitesse maximale de translation $(\mathrm{m} / \mathrm{s})$ & 3,8 \\
\hline Force récupératrice maximale $\left(\mathrm{F}_{\text {gene }}\right)(\mathrm{N})$ & 95,7 \\
\hline Puissance moyenne mécanique $\left(\mathrm{F}_{\text {gene }} \mathrm{V}_{\mathrm{max}}\right)(\mathrm{W})$ & 181
\end{tabular}

\section{Références}

[1] Garcia Burrel I, "Modélisation, commande et optimisation d'un système de micro-cogénération par moteur Stirling "double effet" et générateur asynchrone linéaire", thèse de doctorat ENS Cachan 2007.

[2] François $\mathrm{P}$, “Contribution à la modélisation électromagnétique d'un générateur linéaire à induction appliquée à un micro-cogénérateur Stirling à piston libre", thèse de doctorat, ENS Cachan, 2011

[3] K. Deb et R. Agrawal, “Simulated binary crossover for continuoussearch space”. Complex Systems, volume 9, no. 2, pp. 115-148, 1995.

[4] K. Deb, A. Prata R. Agrawal, “A Fast and Elitist Genetic Algorithm: NSGAII”. IEEE Transactions on evolutionary computation, Volume 6, No. 2, pp. 182-197, April 2002.

[5] I. Boldea, S.A. Nasar, "Linear Electric Actuators and Generators",(Book style) Cambridge University Press 1997.

[6] Y. Mori, S. Torii, D. Ebihara, "End effect Analysis of LinearInduction Motor Based on the Wavelet Transform Technique",IEEE Transaction on Magnetics, Vol.35, No.5, 1999.

[7] Dang T.T, "Theoretical and experimental results ofTubular Linear Induction Generator for StirlingCogenerator System”, ICEM Roma, 6-8 Sept. 2010.

[8] Regnier J, "Conception de systèmes hétérogènes en Génie Électrique par optimisation évolutionnaire multicritère", thèse de doctorat, INP Toulouse 2003

[9] http://www.irf.com/indexnsw.html (Fiches IRGB4059DPbF, IRGB4045DPbF, IRGI4060DPbF, IRGI4061DPbFIRGB4056DPbF) 